\title{
Studies on the Human Prostatic Cancer Cell Line LNCaP
}

\author{
Jos Veldscholte, ${ }^{\star}$ Cor A. Berrevoets and Eppo Mulder \\ Department of Endocrinology and Reproduction, Erasmus University Rotterdam, P.O. Box 1738, \\ 3000 DR Rotterdam, The Netherlands
}

The effects of androgens, antiandrogens, and other steroid hormones on growth of the human prostate cancer cell line LNCaP were studied. Despite the absence of receptors for progesterone and estradiol, the growth rate of the androgen responsive LNCaP-FGC cells increased when cultured in the presence of either estrogens or progestagens. In addition, most antiandrogens were also growth stimulators. This aberrant response was due to a threonine to alanine substitution at amino acid position 868 in the steroid binding domain of the androgen receptor (AR). Only the antiandrogen ICI 176334 could block transcription and cell growth by the mutant receptor. By immunoprecipitation of the AR from LNCaP cells with the specific antibody F39.4.1 and Western blotting, three types of heat-shock proteins co-precipitated: hsp90, hsp70 and hsp56. This co-isolation could be prevented by pre-incubating the cells with androgens or with the antiandrogen hydroxyflutamide. Only the antiandrogen ICI 176334 could block the effect of androgens on complex dissociation and prevent tight nuclear binding of the AR. Hydroxyflutamide could only inhibit tight nuclear binding of the wild-type AR. Therefore, in LNCaP cells the mutation in the steroid binding domain of the AR prevents a blockade of receptor function by most antiandrogens, but not by ICI 176 334, probably because of a different mechanism by which this compound blocks receptor function.

F. Steroid Biochem. Molec. Biol., Vol. 49, No. 4-6, pp. 341-346, 1994

THE LNCAP CELL AS A MODEL SYSTEM TO TO STUDY ANDROGEN RESPONSIVENESS: THE IMPACT OF THE MUTATED ANDROGEN RECEPTOR IN LNCaP CELLS ON RESEARCH RESULTS

Since the establishment of the prostate tumour cell line LNCaP [1], this cell line has been used as an in vitro model for androgen responsive growth (proliferation) of epithelial prostate cancer cells (for a review on the origin of LNCaP and its sublines, see Ref. [2]). The subline used in our studies is the FGC (Fast Growing Colony) subline, which is androgen dependent for growth. A culture which has been passaged for an additional 50 rounds has lost its dependency of, but is still responsive to androgens. The growth of prostate cancer cells is initially highly dependent upon androgen action, and it is of utmost importance that the androgen response system in a model system for prostate cancer is mechanistically similar to that in normal prostate or prostate tumour epithelium cells. For LNCaP cells

Proceedings of the XVI Meeting of the International Study Group for Steroid Hormones, Vienna, Austria, 28 Nov.-1 Dec. 1993. *Correspondence to J. Veldscholte. this is not the case. Despite the absence of estrogen receptors (ER) and progesterone receptors (PR) in LNCaP cells [3], growth of these cells is not only enhanced by androgens, but also by estradiol and progesterone $[1,3,4]$. Furthermore, antiandrogens showed stimulatory rather than inhibiting effects [5]. In our laboratory, detailed studies on the steroid binding specificity of both cytosolic and nuclear androgen receptor (AR) were performed [6]. It was shown that a mutation in the steroid binding domain of the AR of LNCaP cells, resulting in the replacement of a threonine residue at amino acid position 868 by an alanine residue, is responsible for a changed steroid binding specificity. Moreover, in transfection experiments, the mutant, but not the wild-type receptor, enhanced transcription of an androgen responsive reporter gene in response to progestins, estrogens, and some antiandrogens [7].

The original karyotype of LNCaP cells (nearly tetraploid) is well preserved among the sublines [8]. Therefore, the duplication of the $\mathrm{X}$ chromosome, which carries the AR gene [9], may also have occurred in the parental subline. Only the mutated form of the AR was found after genomic sequencing [7], and one 
of the early passages (passage 20) also contains the mutant receptor [10]. Therefore, it is likely that all sublines are homozygous for the mutation. In contrast to the antiandrogens cyproterone acetate (CPA), anandron, and hydroxyflutamide (HF), the antiandrogen ICI 176334 did not stimulate the proliferation rate of LNCaP cells, nor did it enhance transcription from the androgen responsive chloramphenicol acetyltransferase (CAT)-gene in HeLa cells [11] or COS cells [12] transfected with the cDNA of the mutant AR. This suggests that the mechanism of action of ICI 176334 might be different from the mechanism(s) of action of the other antiandrogens. In steroid-free medium, the AR of LNCaP cells is not tightly bound to nuclear components and after rupture of the cells recovered in the cytosol fraction, in association with other proteins. Binding of hormone to these cytosolic receptor complexes induces dissociation of the other proteins. When LNCaP cells were incubated in the absence of hormones, and subsequently the AR-complexes were purified from the cytosol with a specific antibody, heat-shock proteins (hsp90, hsp70, and hsp56) were co-precipitated together with the receptor. Incubation of the cells with either the synthetic androgen R 1881 or with the antiandrogen HF resulted in loss of hsp's co-precipitating with the AR. Incubation of the cells with ICI 176 334, however, did not result in a loss of receptor-associated hsp's, and even blocked the effect of R1881 [11]. The results suggest that R1881 and the antiandrogen $\mathrm{HF}$, in agreement with their agonistic effects on the AR in LNCaP cells, induce receptor-hsp complex dissociation. ICI 176334 blocks this process and therefore acts as an antagonist of androgen action. Also in COS cells expressing either the wild-type or mutant receptor, tight nuclear binding of both types of receptors are blocked by ICI 176334 . HF only blocks tight nuclear binding of the wild-type receptor [12].

In studies by Miller et al. [13] 1,25 dihydroxyvitamin $\mathrm{D}_{3}\left(\mathrm{VD}_{3}\right)$ stimulated the growth rate of LNCaP cells. In contrast to this, we did not observe a stimulation of growth by $\mathrm{VD}_{3}$ [14]. In agreement with Skowronski et al. [15], we found an inhibitory effect of $\mathrm{VD}_{3}$ on androgen induced $\mathrm{LNCaP}$ cell proliferation and in addition we did not observe binding of $\mathrm{VD}_{3}$ to the mutant AR [14]. Therefore, $\mathrm{VD}_{3}$ can neither substitute for androgens, nor for antiandrogens, but affects LNCaP cell proliferation by a separate signal transduction pathway.

\section{ANDROGEN EFFECTS ON LNCAP CELLS: BIPHASIC RESPONSES}

\section{Growth rate of $L N C a P$ cells}

The stimulatory effect of androgens, estrogens, progestins, and antiandrogens on growth of LNCaP cells shows a biphasic dose-response relationship when the cells are cultured in medium with dextran-

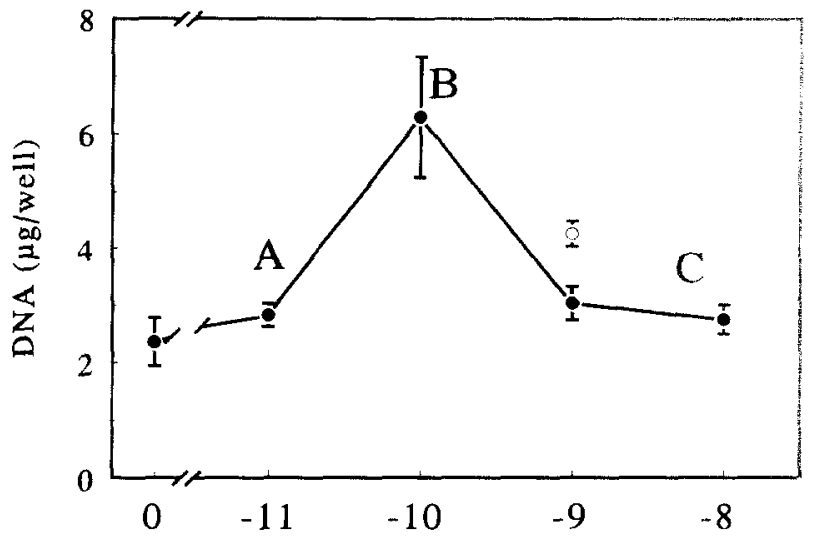

$\mathrm{R} 1881(\log \mathrm{M})$

Fig. 1. Effects of the synthetic androgen R1881 (O) and R1881 + ICI $176334\left(1 \times 10^{-6} \mathrm{M}\right)(O)$ on growth of LNCaP cells during a 6 day culture period. Means and standard deviations of four measurements are shown. (Partly from Ref. [11].)

charcoal-stripped serum $[3,5,16-18]$. Up to a certain optimal concentration, which is different for each ligand, the growth rate increases with increasing concentrations. At concentrations higher than this optimum, growth rate is stimulated to a much lesser extent (see Fig. 1). After culture for several weeks in medium with elevated androgen concentrations, the biphasic effect was lost, but could be re-introduced by changes of medium [19]. The molecular mechanism of the biphasic effect is not understood. It is important to know that the AR is involved and that submaximal stimulation is not the result of a non-specific effect of high concentrations of ligand. This is concluded from the observation that ICI 176334 , the only antiandrogen tested which shows antagonistic effects in LN$\mathrm{CaP}$ cells, is able to antagonize the effect of a supraoptimal concentration of androgens. ICI 176334 competes with androgens for receptor occupation and reduces the actual amount of AR occupied with androgen. As a result, growth rate in the presence of a mixture of ICI 176334 and androgens increased compared to androgens alone (Fig. 1, at $10^{-9} \mathrm{M}$ R1881).

In some studies, the agonistic effects of some antiandrogens on LNCaP cell growth were either not recognized, or were explained by effects not mediated by the AR $[16,20]$. In the report by Olea et al. [16] antagonistic actions of several antiandrogens on LNCaP cell growth were claimed because these antiandrogens inhibited the growth stimulating effect of dihydrotestosterone (DHT). An alternative explanation could be that this apparent antagonistic effect of antiandrogens is due to the use of very high concentrations of DHT (Fig. 1, range B to C). Therefore, the addition of the antiandrogens may have resulted in an increase in the total amount of agonist present, and consequently may have led to a shift in the biphasic 
dose-response curve to a point with lower growth stimulating potency (comparable with a shift from $B$ to $\mathrm{C}$ in Fig. 1). A similar effect could be expected from any combination of compounds with agonistic properties for LNCaP cells. Indeed, DHT inhibited the growth induced by antiandrogens [16]. Sonnenschein and co-workers $[16,21]$ theorized that the stimulating effects of androgens, and also of estrogens, progestins, and antiandrogens, on LNCaP cell proliferation, are mediated by binding of these compounds to, and inhibition of the action of, serum factors with proliferation inhibitory activity. We showed, however, that androgens, estrogens, progestins, and antiandrogens can activate transcription in $\mathrm{HeLa}$ cells through the mutant 'LNCaP AR' [7]. The transcription activation correlated very well with the effects of these compounds on growth rate of $\mathrm{LNCaP}$ cells. The simplest explanation, therefore, is that stimulation of proliferation of LNCaP cells by these non-androgenic compounds is mediated through the AR in these cells: it seems that the postulated serum factors $[16,21]$ may not play a significant role in the aberrant responses of LNCaP cells to non-androgens.

\section{Other AR dependent effects in LNCaP cells}

In addition to effects on growth, there are other processes in LNCaP cells which depend on AR action. The induction of epidermal growth factor receptor, the production of apolipoprotein $\mathrm{D}$, and the secretion of the prostate marker prostatic acid phosphatase (PAP), are not only regulated by androgens, but also by progestins, estrogens, and antiandrogens. The dose-response curves of these effects are also biphasic $[3,17,19,22]$. As for growth rate, this biphasic response pattern also disappeared when the cells were cultured for a few weeks in medium with an increased androgen concentration [19]. For another prostate tumour marker, prostate specific antigen (PSA), no biphasic dose-response curve was found. Its mRNA levels in LNCaP cells and the levels of secretion are both elevated in a monophasic mode by androgens and non-androgens $[19,22-24]$. In agreement with this, HF stimulated PSA mRNA levels [22]. In contrast, this compound could partially decrease DHT-induced PSA mRNA levels in another study [23]. In LNCaP cells, androgens also decrease AR mRNA levels, while increasing $A R$ protein levels. AR mRNA levels were decreased by testosterone, R1881, the synthetic androgen mibolerone, estradiol, progesterone, and CPA [24-26].

\section{In conclusion}

In LNCaP cells, several androgen dependent effects are also elicited by estrogens, progestins, and some antiandrogens. Many of the responses to these compounds showed a characteristic biphasic pattern. In some cases, antagonistic effects of antiandrogens were claimed, but these effects may in fact represent agonistic effects. Misinterpretations can occur when a compound is tested at only one concentration, especially when a biphasic pattern of stimulation or repression is concerned. Rapid changes of the androgen sensitivity occur under the influence of culture conditions, resulting in a loss of the biphasic doseresponse. These changes are partly reversible and most likely due to an adaptation of the cells.

\section{BY WHAT MECHANISM DID THE ANTIANDROGENS BECOME AGONISTS FOR THE MUTATED RECEPTOR?}

\section{The role of dissociation rate}

It has been suggested that fast dissociation of a steroid receptor ligand would explain the antagonistic properties of this compound [27]. For a number of derivatives of the antiandrogen $\mathrm{HF}$, however, no strict correlation was found between dissociation rate and biological potency [28]. This indicates that, even if dissociation of a compound plays a role in the antagonistic action of some compounds, it is not the only existing mechanism of antagonism.

If the antagonistic action of a certain compound is elicited by its fast dissociation from the receptor, then at least one irreversible step in receptor transformation is required, because otherwise a high concentration of the compound would drive the receptor towards transcription activation, in which case this compound would be an agonist. Therefore, if a fast ligand dissociation plays a role in antagonistic action, it is not the dissociation per se which blocks receptor action, but the condition in which the receptor is left unliganded.

\section{Antagonists may act at different steps in the transform- ation cascade}

There are several steps in the cascade of receptor transformation which may be blocked by antagonists (Fig. 2). These blockades can theoretically be the result of either a high rate of dissociation of the antagonist and a subsequent irreversible process (as described in the previous paragraph), or an aberrant interaction of an antagonist with the receptor, without dissociation of that compound from the receptor. In addition, more than one of the steps shown in Fig. 2 may be involved in the inhibitory actions of antagonists. There are three possible explanations for the agonistic actions of CPA, $\mathrm{HF}$, and anandron on the mutant receptor (hypothesis $A$ to $\mathrm{C}$ in Fig. 2).

(1) In the first hypothesis, a blockade of receptor-hsp dissociation by these compounds, but not the one induced by ICI 176 334, is overthrown by the mutation. A comparison of the wild-type receptor and the mutant receptor with respect to antiandrogenmediated receptor-hsp complex dissociation will be necessary to test this theory experimentally.

(2) Since in LNCaP cells ICI 176334 blocks the dissociation of the receptor-hsp complex and acts as an 


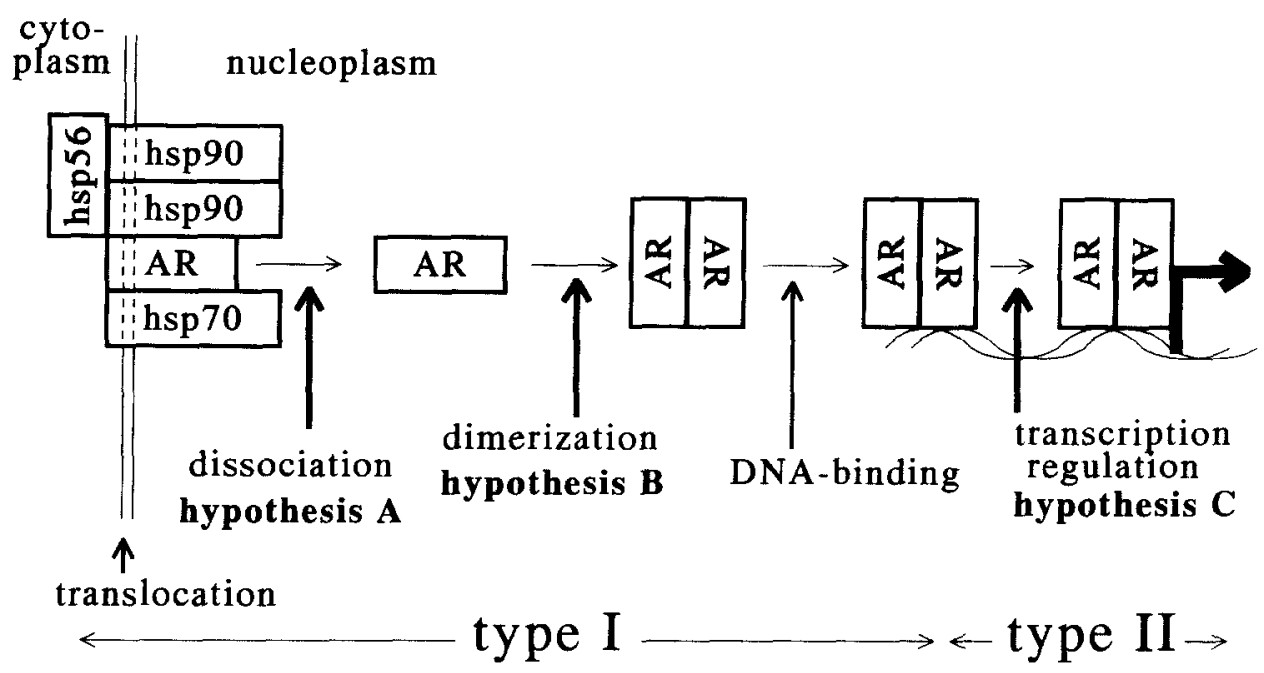

Fig. 2. Several steps in the cascade of receptor transformation which are possibly blocked by antagonists. In hypothesis $\mathrm{A}$ through $\mathrm{C}$, antagonists have different effects. Type $\mathrm{I}$ antagonists block receptor action before DNA-binding occurs and type II antagonists block receptor action following DNA-binding.

antagonist for both the wild-type and mutant receptor [11], it is conceivable that the mutation has not altered receptor-hsp interactions. Therefore, CPA, HF, and anandron possibly block a receptor transformation step which succeeds hsp complex dissociation, and this block might be eliminated by the mutation. The LNCaP mutation at amino acid position 868 is located in the so-called heptad repeat region. It contains a heptad repeat of hydrophobic amino acid residues, which is highly conserved among the steroid/thyroid hormone receptor superfamily and has been suggested to be involved in dimerization of the ER [29]. It seems possible, therefore, that the dimerization step is normally blocked by CPA, HF, and anandron, but that this blocking effect is lost by the mutation (hypothesis B in Fig. 2). Dimerization is necessary for binding to DNA to occur. This can be detected by the presence of tight nuclear bound receptors. In favour of hypothesis B it was observed that the amount of tightly nuclear bound receptors was increased by CPA and $\mathrm{HF}$ for the mutant receptor when compared with the wild-type receptor [12].

(3) A third possibility (hypothesis C in Fig. 2) is that antiandrogens still allow receptor dimerization and transformation of the receptor to a DNAbinding state. In addition, only in the case of a mutant AR like in LNCaP cells, the ligand-receptor complex activates transcription through a C-terminally located, ligand-dependent transcription activation function (TAF, see Fig. 3). This TAF (like TAF-2 from ER and PR [31-33]) is activated only by binding of an agonist to the wild-type receptor, and is thus ligand dependent. In hypothesis C, CPA, due to the mutation, has an activating effect on a hormone-dependent TAF (Fig. 2). An additional argument in favour of this hypothesis is that for the ER, TAF-2 has been described as a small conserved stretch of amino acid residues, important for ligandmediated transcription activation [34]. The homologous stretch in the AR, amino acid residues 884-891, is very close to the mutation at position 868 in the $\mathrm{AR}$ in LNCaP cells. It can be envisaged that the mutation has changed the conformation in this region, in a way that allows antiandrogen-mediated transcription activation through the C-terminal TAF (see Fig. 3).

Antagonists which block the binding of the PR to DNA were called type I antagonists. The antagonists which allow binding of the receptor to the hormone response element, but fail to activate the hormonedependent TAF2 (the ligand-dependent transcription activation function present in the C-terminal domain of the receptor), were called type II antagonists [35] The reversed typification was used by others $[31,36]$. When the first terminology is used for AR antagonists, then-at least in LNCaP cells-ICI 176334 is a type I antagonist. CPA possibly is a type II antagonist (Fig. 2). HF is a type I antagonist because it does not induce tight nuclear binding of the wild-type AR in COS cells [12].

\section{PRACTICAL IMPLICATIONS}

The use of the mutated AR of LNCaP cells in the studies described here, provided a useful tool to obtain more insight into the mechanisms of inhibition of androgen action by antiandrogens. The various antiandrogens showed differences in their mechanisms of action, and therefore it can be envisaged that in the treatment of androgen-dependent disorders, these compounds also show differences in effectiveness and side effects. Cell and promoter context specific regulation of gene transcription might play an important role in the mechanisms underlying the effects 
wild-type

hdTP
LNCaP mutant

warge
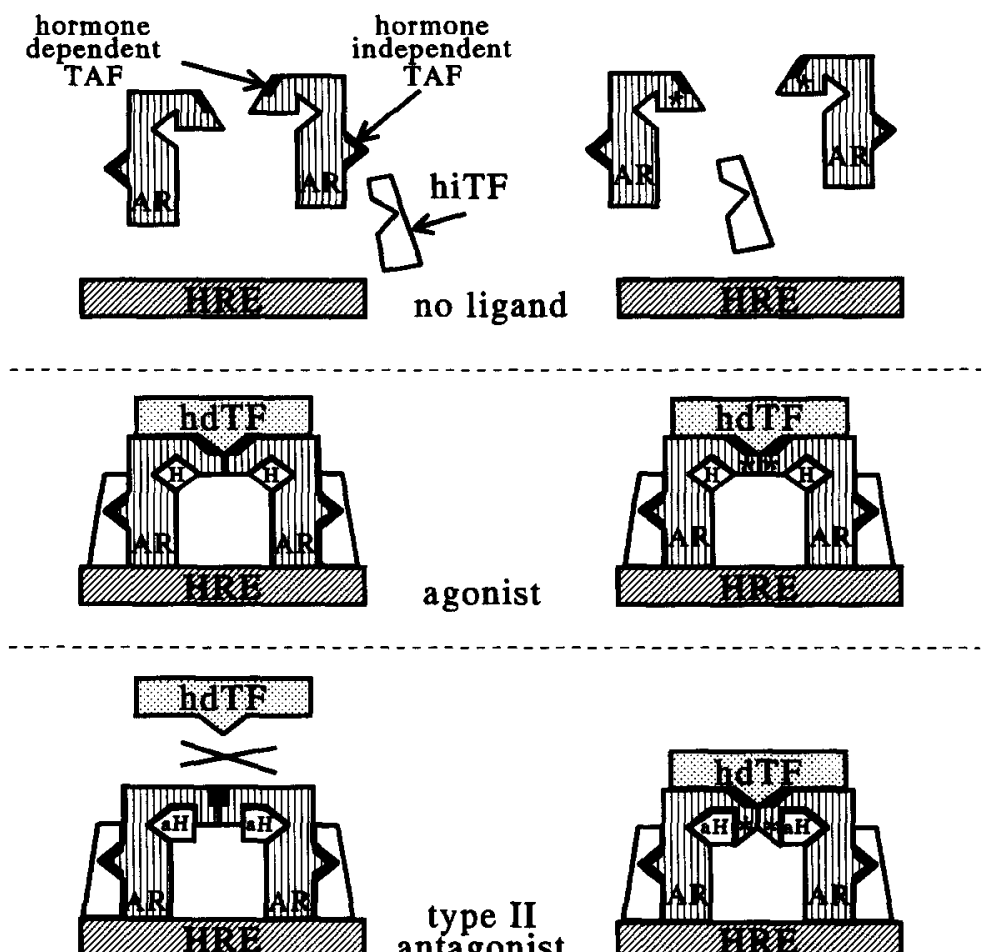

type II antagonist

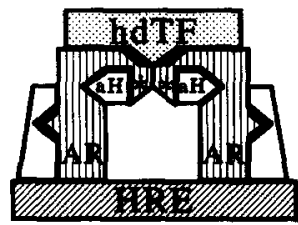

Fig. 3. The putative effects of the mutation in the AR of LNCaP cells on the actions of agonists and type II antagonists. For clarity, hsps have not been included in the figure. In both wild-type and mutant receptor, hormones $(\mathbf{H})$ induce a change in the receptor molecule (AR) that allows dimerization and binding of the dimer to the HRE. In addition, the ligand induces a change in conformation which allows the hormonedependent TAF to interact with a transcription factor (hdTF), resulting in transcription activation. In the wild-type receptor, type II antagonists $(\mathrm{aH})$ induce dimerization and binding of the dimer to the HRE, but the interaction with hdTF is inhibited. Whether or not transcription occurs, is now dependent upon the presence of a second type of transcription factor (hiTF) (and possibly promoter context) which can interact with a hormone-independent TAF. The hiTF in this model is not present in HeLa cells and LNCaP cells but is present in COS and CV-1 cells. In the mutant receptor, the mutation $\left(^{\star}\right)$ resulted in a changed response to type II antagonists. Despite the occupancy of the receptor by the antagonist, the hormone-dependent TAF is able to interact with a hdTF transcription factor, resulting in transcription activation.

of antiandrogens in a complex organism. More knowledge about the relation between the structure of antagonists and their modes of action may be very helpful in the design of new steroid hormone receptor antagonists.

Acknowledgement-This study was supported by the Dutch Cancer Society (NKB) through Grant IKR 90-13.

\section{REFERENCES}

1. Horoszewicz J. S., Lying S. S., Kawinski E., Karr J. P., Rosenthal H., Chu T. M., Mirand E. A. and Murphy G. P. LNCaP model of human prostatic carcinoma. Cancer Res. 43 (1983) 1809-1818.

2. Van Steenbrugge G. J., Van Uffelen C. J. C., Bolt J. and Schröder F. H.: The human prostatic cancer cell line LNCaP and its derived sublines-An in vitro model for the study of androgen sensitivity. F. Steroid Biochem. Molec. Biol. 40 (1991) 207-214.

3. Schuurmans A. L. G., Bolt J., Voorhorst M., Blankenstein R. A. and Mulder E.: Regulation of growth and epidermal growth factor receptor levels of LNCaP prostate tumor cells by different steroids. Int. F. Cancer 42 (1988) 917-922.

4. Schulz P., Bauer H. W. and Fittler F.: Steroid hormone regulation of prostatic acid phosphatase expression in cultured human prostatic carcinoma cells. Biol. Chem. Hoppe-Seyler 336 (1985) 1033-1039.

5. Wilding G., Chen $M$. and Gelmann E. P.: Aberrant response in vitro of hormone-responsive prostate cancer cells to antiandrogens. Prostate 14 (1989) 103-115.

6. Veldscholte J., Voorhorst-Ogink M. M., Bolt-De Vries J., Van Rooij H. C. J., Trapman J. and Mulder E.: Unusual specificity of the androgen receptor in the human prostate tumor cell line $\mathrm{LNCaP}-$ high affinity for progestagenic and estrogenic steroids. Biochim. Biophys. Acta 1052 (1990) 187-194.

7. Veldscholte J., Ris-Stalpers C., Kuiper G. G. J. M., Jenster G., Berrevoets C., Claassen E., Van Rooij H. C. J., Trapman J., Brinkmann A. O. and Mulder E.: A mutation in the ligand binding domain of the androgen receptor of human LNCaP cells affects steroid binding characteristics and response to antiandrogens. Biochem. Biophys. Res. Commun. 173 (1990) 534-540.

8. König J. J., Kamst E., Hagemeyer A., Romijn J. C., Horoszewicz J. and Schröder F. H.: Cytogenetic characterization of several androgen responsive and unresponsive sublines of 
the human prostatic carcinoma cell line LNCaP. Urol. Res. 17 (1989) 79-86.

9. Trapman J., Klaassen P., Kuiper G. G. J. M., Van der Korput J. A. G. M., Faber P. W., Van Rooii H. C. J., Geurts van Kessel A., Voorhorst M. M., Mulder E. and Brinkman A. O.: Cloning, structure and expression of a $\mathrm{LNCaP}$ encoding the human androgen receptor. Biochem. Biophys. Res. Commun. 153 (1988) 241-248.

10. C. Ris-Stalpers, unpublished results.

11. Veldscholte J., Berrevoets C. A., Brinkmann A. O., Grootegoed J. A. and Mulder E.: Antiandrogens and the mutated androgen receptor of LNCaP cells-Differential effects on binding affinity, heat-shock protein interaction and transcription activation. Biochemistry 31 (1992) 2393-2399.

12. Berrevoets C. A., Veldscholte J. and Mulder E.: Effects of antiandrogens on transformation and transcription activation of wild-type and mutated (LNCaP) androgen receptors. F. Steroid Biochem. Molec. Biol. 46 (1993) 731-736.

13. Miller G. J., Stapleton G. E., Ferrara J. A., Lucia M. S., Pfister S., Hedlund T. E. and Upadhya P.: The human prostatic carcinoma cell line LNCaP expresses biologically active, specific receptors for 1 alpha,25-dihydroxyvitamin D3. Cancer Res. 52 (1992) 515-520

14. Van Steenbrugge et al. (unpublished data).

15. Skowronski R. J., Peehl D. M. and Feldman D.: Vitamin D and prostate cancer: 1,25 dihydroxyvitamin $\mathrm{D} 3$ receptors and actions in human prostrate cancer cell lines. Endocrinology 132 (1993) 1952-1960.

16. Olea N., Sakabe K., Soto A. M. and Sonnenschein C.: The proliferative effect of anti-androgens on the androgen-sensitive human prostate tumor cell line LNCaP. Endocrinology 126 (1990) 1457-1463.

17. Simard J., Veilleux R., De Launoit Y., Haagensen D. E. and Labrie F.: Stimulation of Apolipoprotein-D secretion by steroids coincides with inhibition of cell proliferation in human I.NCaP prostate cells. Cancer Res. 51 (1991) 4336-4341.

18. De Launoit Y., Veilleux R., Dufour M., Simard J. and Labric $F$.: Characteristics of the biphasic action of androgens and of the potent antiproliferative effects of the new pure antiestrogen EM-139 on cell cycle kinetic parameters in LNCaP human prostatic cancer cells. Cancer Res. 51 (1991) 5165-5170.

19. Langeler E. G., Van Uffelen C. J. C., Blankenstein M. A., Van Steenbrugge G. J. and Mulder E.: Effect of culture conditions on androgen sensitivity of the human prostatic cancer cell line LNCaP. Prostate 23 (1993) 213-223.

20. Wolf D. A., Schulz P. and Fittler F.: Synthetic androgens suppress the transformed phenotype in the human prostate carcinoma cell line LNCaP. Br. F. Cancer 64 (1991) 47-53.

21. Sonnenschein C., Olea N., Pasanen M. E. and Soto A. M.: Negative controls of cell proliferation - human prostate cancer cells and androgens. Cancer Res. 49 (1989) 3474-3481.

22. Henttu P. and Vihko P.: Steroids inversely affect the biosynthesis and secretion of human prostatic acid phosphatase and prostate-specific antigen in the LNCaP cell line. F. Steroid. Biochem. Molec. Biol. 41 (1992) 349-360.

23. Young C. Y.-F., Montgomery B. T., Andrew's P. E., Qiu S.,
Bilhartz D. L. and Tindall 1). 1.: Hormunat fezulaten: prostate-specific antigen messenger $\mathrm{RNA}$ in human pristat adenocarcinoma cell line INCal'. Cancer Re sy cy 3748-3752.

24. Henttu P., Liao S. and Vihko P': Androgens up egulate th human prostate-specific antigen messenger ribonucleic acis (mRNA), but down-regulate the prostatic acid phosphatas mRNA in the INCaP cell line. Endorinology 130 $766-772$

25. Quarmby V. E., Yarbrough W. G., Lubahn D. B. French 1and Wilson E. M:: Autologous down-regulation of androgen receptor messenger ribonucleic acid. Molec. Endorr. 4990 , 22-28

26. Krongrad A., Wilson C. M., Wilson J. D., Allman I), K and McPhaul M. J.: Androgen increases androgen receptor protein while decreasing receptor messenger RNA in I.NCaP cells Molec. Cell. Endocr. 76 (1991) 79-88.

27. Raynaud J. P., Bouton M. M., Moguilewsky M. Ojasoo T. Philibert D., Beck G., Labrie F. and Mornon J. P.: Steroid hormone receptors and pharmacology. 7. Steroid Biochem. 12 (1980) 143-157.

28. Wakeling A. E., Furr B. I. A., Glen A. 'T and Hughes L. R Receptor binding and biological activity of steroidal and non steroidal antiandrogens. F. Steroid Biochem. 15 (1981) 355-359

29. Fawell S. E., Lees J. A., White R. and Parker M. G.: Characterization and colocalization of steroid binding and dimerization activities in the mouse estrogen receptor. Cell $60(1990) 953-962$

30. Simental J. A., Sar M., Lane M. V., French F. S. and Wilson F. M.: Transcriptional activation and nuclear targeting signal: of the human androgen receptor. 7. Biol. Chem. 266 1991 $510-518$.

31. Gronemeyer H., Benhamou B., Berry M., Bocquel M. T., Gofflo 1)., Garcia T., Lerouge T., Metzger D., Meyer M. E., Tora I. Vergezac A. and Chambon P.: Mechanisms of antihormonc action. F. Steroid Biochem. Molec. Biol. 41 (1992) 217-221.

32. Green S.: Modulation of oestrogen receptor activity by oestro gens and anti-oestrogens. F. Steroid Biochem. Molec. Biol. 37 (1990) 747-751

33. Klein-Hitpass I.,, Tsai S. Y., Weigel N. I., Allan G. F., Riley D., Rodriguez R., Schräder W. T., Tsai M.-J. and O'Malley B. W.: The progesterone receptor stimulates cell-free transcription by enhancing the formation of a stable preinitiation complex. Cell 60 (1990) 247-257.

34. Danielian P. S., White R., L.ees J, A. and Parker M. G Identification of a conserved region for hormone dependent transcriptional activation by steroid hormone receptors. $E M B O$ fl 11 (1992) 1025-1033.

35. Klein-Hitpass L., Cato A. C. B., Henderson D. and Ryffe G. U.: Two types of antiprogestins identified by their differential action in transcriptionally active extracts from T47D cells. Nucleic Acids Res. 19 (1991) 1227-1234.

36. Reesc J. C. and Katzenellenbogen B. S.: Differential DNA binding abilities of estrogen receptor occupied with two classe: of antiestrogens--studies using human estrogen receptor overexpressed in mammalian cells. Nucleic Acids Res. 19 (1991 $6595-6602$. 